\title{
MYC expression promotes lipid metabolism and metabolic plasticity in human mammary epithelial cell
}

JESSICA C. CASCIANO, ADAM COHEN-NOWAK, JOHAN VANDE VOORDE, QIFENG ZHANG, SUSAN CHALMERS*, MAIRI SANDISON*, ANN HEDLEY, TONY MCBRYAN, THOMAS BEER, HSIN-YAO TANG, DAVID W. SPEICHER, PETER ADAMS, XIUFENG LIU, RICHARD SCHLEGEL, JOHN MCCARRON*, MICHAEL J. WAKELAM, EYAL GOTTLIEB, ZACHARY T. SCHUG,

*University of Strathclyde, United Kingdom

MYC is one of the most commonly mutated and highly amplified oncogenes in human breast cancer. MYC amplifications occur most frequently in triple-negative breast cancers (TNBCs). TNBCs can be divided into two molecular subtypes: basal-like and claudin-low breast cancers. These cancers tend to be extremely aggressive and are strongly associated with disease recurrence, poor prognosis and high mortality. In particular, claudin-low tumors are classified by a loss of tight junctions and cell-tocell contacts and an enrichment for genes associated with an epithelial-to-mesenchymal transition (EMT) and mammary stem cells (also known as tumor-initiating cells). Despite the high level of disease severity, there are no targeted therapies for claudin-low TNBCs. To address this unmet need, we utilized human mammary epithelial cells (HuMECs) that express oncogenic levels of MYC and a mutant MYC (T58A) to characterize the behavioral and metabolic changes that occur during the formation of MYC-driven breast cancers. We found that MYC regulates the expression of genes associated with cell stemness, EMT, lipid metabolism, and calcium ( $\mathrm{Ca} 2+)$ signaling and that the expression of this gene signature promotes cell growth, survival, migration, and metabolic plasticity. The gene signature of MYC-expressing HuMECs highly correlates with the gene signature of claudinlow breast cancers, therefore highlighting the relevance of our HUMEC model to human claudin-low breast cancer. We found the major drivers underlying the MYC-dependent changes in cell behavior to be stimulation of $\mathrm{Ca} 2+$ signaling and strong activation of lipid metabolism. $\mathrm{Ca} 2+$ signaling is stimulated through the MYC-dependent repression of $\mathrm{Ca} 2+$ efflux mechanisms; elevated cytosolic $\mathrm{Ca} 2+$ then consequently stimulates a Ca2+/calmodulin kinase kinase 2 (CAMKK2)/AMPK signaling axis that activates fatty acid scavenging and transport, as well as $\beta$-oxidation. Enhanced lipid metabolism thereby provides the necessary biomass (fatty acids) for phospholipid biosynthesis and energy (ATP) to support the metabolically demanding processes of cell growth, proliferation, and migration. In all, our findings provide a strong rationale for targeting lipid metabolism and the Ca2+/CAMKK2/AMPK signaling axis in MYC-driven, and potentially claudin-low, breast cancers.

Keywords: MYC, triple-negative breast cancers (TNBCs), human mammary epithelial cells (HuMECs)

Accepted manuscript of the following research output: Casciano, J. C., Cohen-Nowak, A., Vande Voorde, J., Zhang, Q., Chalmers, S., Sandison, M., ... Schug, Z. T. (2018). MYC expression promotes lipid metabolism and metabolic plasticity in human mammary epithelial cell. Cancer Research, $78(13$ Suppl). https://doi.org/10.1158/1538-7445.AM2018-1441. 